\title{
Mitochondrial dysfunction is induced by the overexpression of UCP4 in 3T3-L1 adipocytes
}

\author{
CHUN-LIN GAO ${ }^{1,2,3^{*}}$, JIN-GAI ZHU $^{1,3^{*}}$, YA-PING ZHAO ${ }^{1,3}$, XIAO-HUI CHEN $^{1,3}$, CHEN-BO JI $^{1,3}$, \\ CHUN-MEI ZHANG ${ }^{1,3}$, CHUN ZHU ${ }^{1,3}$, ZHENG-KUN XIA ${ }^{2}$, YU-ZHU PENG ${ }^{1}$ and XI-RONG GUO ${ }^{1,3}$ \\ ${ }^{1}$ Department of Pediatrics, Nanjing Maternal and Child Health Hospital of Nanjing Medical University, Nanjing 210004; \\ ${ }^{2}$ Department of Pediatrics, Jinling Hospital, Nanjing 210002; ${ }^{3}$ Institute of Pediatrics, \\ Nanjing Medical University, Nanjing 210029, P.R. China
}

Received August 31, 2009; Accepted October 5, 2009

DOI: 10.3892/ijmm_00000315

\begin{abstract}
Uncoupling proteins (UCPs) belong to a superfamily of mitochondrial transporters that uncouple ATP synthesis from electron transport. We have previously shown that uncoupling protein 4 (UCP4) is differentially expressed in omental adipose tissue in diet-induced obese and normal rats. Overexpression of UCP4 promotes proliferation and inhibits apoptosis and differentiation of preadipocytes. In this work, we further characterized the effect of UCP4 on mitochondrial function in mature 3T3-L1 adipocytes. Transmission electron microscopy (TEM) showed that adipocytes overexpressing UCP4 displayed condensed mitochondria with twisted, condensed, and unclear cristae. Moreover, the loss of the mitochondrial membrane potential and intramitochondrial calcium was found. The adipocytes overexpressing UCP4 also showed decreased mitochondrial copy number (mtDNA) and lower mRNA expression of key factors in mitochondrial biogenesis, including PGC- $1 \alpha$ and mtTFA. NRF-1 and ERRß levels were down-regulated, while NRF-2 levels were upregulated. In addition, UCP4 overexpression impaired mitochondrial fusion and fission, as indicated by decreased mitofusin $\mathrm{mfn} 1$, $\mathrm{mfn} 2$, and mitofission DRP1. When it came to total adipocytes, the UCP4 overexpressing adipocytes showed higher production reactive oxygen species and diminished levels of intracellular ATP. Furthermore, overexpression of UCP4 brought about impaired insulin sensitivity in adipocytes. UCP4 plays an important role in mitochondrial function and
\end{abstract}

Correspondence to: Dr Xi-Rong Guo, Institute of Pediatrics, Nanjing Medical University, Nanjing 210029, P.R. China

E-mail:xrguo@njmu.edu.cn

Dr Yu-Zhu Peng, Department of Pediatrics, Nanjing Maternal and Child Health Hospital of Nanjing Medical University, Nanjing 210004, P.R. China

E-mail: pyz1131@hotmail.com

${ }^{*}$ Contributed equally

Key words: uncoupling proteins, mitochondria, 3T3-L1 adipocytes, insulin resistance adipocyte insulin resistance. Its function deserves further attention.

\section{Introduction}

In line with the increasing prevalence of obesity, the incidence of type 2 diabetes mellitus has increased dramatically. Central to the etiology of type 2 diabetes is insulin resistance, an early detectable characteristic preceding the development of type 2 diabetes. Insulin resistance indicates a state of reduced responsiveness of insulin-sensitive tissues to circulatory levels of insulin, i.e., there is reduced glucose disposal and impaired inhibition of hepatic glucose output at a given concentration of insulin (1). Evidence gathered in recent decades has pointed towards an important role for mitochondrial dysfunction in the development of insulin resistance, especially for mitochondrial oxidative capacity in the etiology of type 2 diabetes (2-4). Mitochondria are the major source of reactive oxygen species (ROS) in cells and cumulated evidence points towards a causal role for ROS in the development of insulin resistance (5). ROS and oxidative stress lead to the activation of multiple serine/ threonine kinase signaling cascades (6) including an increase in serine phosphorylation, which decreases the extent of activating tyrosine phosphorylation $(7,8)$. The kinases that are activated by oxidative stress include JNK, p38 MAPK, and IKKB (9-12).

Mitochondrial uncoupling proteins (UCPs) are a subfamily of the mitochondrial transporter family, which are located in the inner mitochondrial membrane and uncouple electron transport from ATP synthesis. Uncoupling protein 4 (UCP4), one of five members recently described, possesses features characteristic of mitochondrial transporter proteins, and its ectopic expression in mammalian cells reduces mitochondrial membrane potential (MMP) (13). PC12 cells expressing UCP4 have attenuated mitochondrial oxidative stress and regulated $\mathrm{Ca}^{2+}$ homeostasis (14). The higher UCP4 levels observed in aged female rats led to better oxidative homeostasis maintenance in the females (15). UCP4 overexpression also remarkably protected neurons against $\mathrm{MPP}^{+}$-induced cell death via the preservation of ATP levels and MMP, and the suppression of oxidative stress (16). While UCP4 is mainly expressed in the brain, we previously showed that UCP4 is differentially expressed in omental adipose tissue in dietinduced obese and normal rats and that overexpression of 
Table 1. Oligonucleotide sequences for primer/probe sets used in TaqMan analysis.

\begin{tabular}{llll}
\hline Gene & Forward primer $\left(5^{\prime}-3^{\prime}\right)$ & Reverse primer $\left(5^{\prime}-3^{\prime}\right)$ & Probe \\
\hline Cyt B & TTTTATCTGCATC & CCACTTCATCTTAC & AGCAATCGTTCACCT \\
& TGAGTTTAATCCT & CATTTATTATCGC & CCTCTCCTCCAC \\
TS & GTGGCGGCCAA & AGGCGTTCAGTCA & CCATTGGCTCCT \\
PGC-1 $\alpha$ & GCGTTCATAG & TAATCCCACAG & \\
mtTFA & TGGAAATCATA & TGAGGACCGCT & \\
& GGAATGCAG & AGCAAGTTG & \\
B-actin & GCGTGCTAAAA & TGCTGGAAAAAC & \\
& CCTGAGGCTC & ACTTCGGAATA & TAGAGGTCTTTAC \\
\hline
\end{tabular}

UCP4 can promote proliferation and inhibit apoptosis and differentiation of preadipocytes (17). Although UCP4 plays a protective role in neurons, little information has been collected on UCP4 function and its effect on mitochondrial function in adipocytes. Thus, further studies are still necessary.

The aim of this study was to determine whether overexpression of UCP4 in 3T3-L1 adipocytes affects adipocyte mitochondrial function parameters, including mitochondrial morphology, mitochondrial DNA (mtDNA) copy number, mitochondrial biogenesis, intracellular ROS levels and ATP levels, intramitochondrial $\mathrm{Ca}^{2+}$ concentration, $\mathrm{MMP}$, and the insulin sensitivity of adipocytes.

We show that overexpression of UCP4 in 3T3-L1 adipocytes induced obvious mitochondrial dysfunction and insulin resistance. Upon UCP4 expression, mitochondrial morphology, biogenesis, and mtDNA copy number were all altered. Increased levels of intracellular ROS, loss of MMP, and intramitochondrial calcium may have contributed to mitochondrial dysfunction.

\section{Materials and methods}

Antibodies. Primary rabbit polyclonal nuclear respiratory factor-1 and -2 (NRF-1 and -2) and mfn2 antibodies were purchased from Abcam (Abcam, MA, USA). Rabbit polyclonal PGC-1ß antibodies were purchased from Lifespan Biosciences. DRP1, SIRT1, and Mfn1 were purchased from Santa Cruz Biotechnology (Santa Cruz, CA, USA). Primary rabbit polyclonal estrogen-related receptor $\beta(E R R B)$ antibodies were purchased from lifespan biosciences (Seattle, WA, USA). B-actin antibody was purchased from Cell Signaling Technology (Danvers, MA, USA). Peroxidase-conjugated AffiniPure goat anti-rabbit secondary antibodies were from Zhongshan Gold Bridge Biotechnology (Beijing, China).

Cell culture and UCP4 transfection. The mouse preadipocyte fibroblast line 3T3-L1 (ATCCCL173) was cultured in Dulbecco's modified Eagle's medium (DMEM) (Gibco BRL, Grand Island, NY, USA) containing $10 \%$ fetal bovine serum (FBS) (Gibco BRL), $100 \mathrm{U} / \mathrm{ml}$ penicillin, and $0.1 \mathrm{mg} / \mathrm{ml}$ streptomycin (Amresco, OH, USA) in $5 \% \mathrm{CO}_{2}$ at $37^{\circ} \mathrm{C}$. Fulllength rat UCP4 cDNAs were amplified by reverse transcriptase PCR (RT-PCR) using mRNAs isolated from white adipose tissues. Primer sequences were, forward, 5'-CGC GGA TCC GCC ACC ATG CCT ATC GCG AG-3', and reverse, 5'-CCC
AAG CTTAAATGG GCT GAC TCC-3', and contained BamHI and HindIII sites, respectively. The PCR products were then subcloned into pcDNA3.1a(-)/His expression vectors. The UCP4 constructs and pcDNA3.1a(-)/His vectors were each transfected separately into 3T3-L1 preadipocytes using Fugene 6 (Roche Molecular Biochemicals, Indianapolis, IN, USA). After $24 \mathrm{~h}$, cells were cultured in selective medium containing $800 \mu \mathrm{g} / \mathrm{ml} \mathrm{G} 418$ (Sigma, St. Louis, MO, USA) for the selection of resistant colonies. Cells were fed with selective medium every 3 days until resistant colonies could be identified. These resistant foci were selected, expanded, tested for expression, and frozen for future experiments. To induce differentiation, 3T3-L1 preadipocytes were grown to confluence for 2 days. Two days later, cells were induced to differentiate (day 0 ) with the addition of a standard differentiation-inducing mix. Insulin $(100 \mathrm{nM})$, dexamethasone $(0.25 \mu \mathrm{M})$ and isobutylmethylxanthine $(0.5 \mathrm{mM})$ (Sigma) were added to the medium for the first $48 \mathrm{~h}$. From days 2 to 4 , the full medium was only supplemented with $100 \mathrm{nM}$ of insulin. Cells were then switched to DMEM containing only $10 \%$ FBS for the remaining days. Cultures were replenished every 2 days.

Glucose uptake. 2-Deoxy-D-[3H] glucose (CIC, Beijing, China) uptake was assayed as described previously but with minor modifications (18). Cells were cultured in six-well plates and induced to differentiate into mature adipocytes. Before the experiment, cells were serum starved for $3 \mathrm{~h}$ in DMEM containing $0.5 \%$ FBS. The cells were then washed twice with phosphate-buffered saline (PBS) and incubated in KRPHEPES buffer (30 mM HEPES pH 7.4), $10 \mathrm{mM} \mathrm{NaHCO}$, $120 \mathrm{mM} \mathrm{NaCl}, 4 \mathrm{Mm} \mathrm{KH}_{2} \mathrm{PO}_{4}, 1 \mathrm{mM} \mathrm{MgSO}$, and $1 \mathrm{mM}$ $\mathrm{CaCl}_{2}$ ) in the presence or absence of $100 \mathrm{nM}$ insulin for $30 \mathrm{~min}$ at $37^{\circ} \mathrm{C}$. Labeled 2-deoxy-D-[3H] glucose was added to a final concentration of $2 \mu \mathrm{Ci} / \mathrm{ml}$. After $10 \mathrm{~min}$ at $37^{\circ} \mathrm{C}$, the reaction was terminated by washing 3 times with ice-cold PBS supplemented with $10 \mathrm{mM}$ D-glucose. Cells were solubilized by the addition of $200 \mu \mathrm{l}$ of $1 \mathrm{M} \mathrm{NaOH}$ to each well, and aliquots of cell lysates were transferred to scintillation vials for radioactivity counting. The remainder of the lysate was used for the protein assay.

Real-time RT-PCR for mitochondrial DNA. Relative amounts of nuclear DNA and mtDNA were determined by quantitative real-time PCR. The ratio of mtDNA to nuclear DNA reflects 
the tissue concentration of mitochondria per cell. For this purpose, a 120-nucleotide-long mtDNA fragment within the CYTB gene was selected for the quantification of mtDNA according to He et al (19).

Quantitative real-time $R T$-PCR. Total RNA from adipocytes was extracted using Trizol reagent (Invitrogen). First-strand cDNA was generated with random primers using a reverse transcription kit (Invitrogen). Real-time analysis was performed on an ABI 7300 RT-PCR system (Foster City, CA, USA) with the SYBR Green kit (Applied Biosystems). Each sample was assayed in duplicate in a $25 \mu 1$ reaction volume containing $2.5 \mu 1 \mathrm{cDNA}, 12.5 \mu 1 \mathrm{SYBR}$ Green master mix (Applied Biosystems), and $0.58 \mu \mathrm{mol} / 1$ of each primer. Negative controls (no template or selected untranscribed RNA) were run to ensure the absence of contamination. Analysis was performed according to the $\Delta \mathrm{Ct}$ method using $ß$-actin as the housekeeping gene. Specific primers for each gene were designed to amplify a single product (see Table I), as confirmed by regular PCR and dissociation curve analysis post-real-time PCR. The primer sequences are available upon request.

Electromicrograph morphometry. The adipocytes were collected after trypsin digestion, dissected, and fixed in a mixture of $2.5 \%$ glutaraldehyde, $1.25 \%$ paraformaldehyde, and $0.03 \%$ picric acid in $0.1 \mathrm{M}$ sodium cacodylate buffer $(\mathrm{pH} 7.4)$. Cells were then washed in $0.1 \mathrm{M}$ cacodylate buffer, postfixed with $1 \%$ osmium tetroxide $/ 1.5 \%$ potassium ferrocyanide for $1 \mathrm{~h}$, washed in water, and stained in $1 \%$ aqueous uranyl acetate for $30 \mathrm{~min}$, followed by dehydration in different concentrations of alcohol ( $5 \mathrm{~min}$ in 70,90 and 100\% alcohol). The samples were then infiltrated and embedded in TAAB Epon (Marivac Canada Inc., St. Laurent, Canada). Ultrathin sections ( 60 nm) were cut on a Reichert Ultracut-S microtome, placed onto copper grids stained with uranyl acetate and lead citrate, and examined in a JEOL 1200EX.

Western blotting. Treated cells were washed with ice-cold PBS and lysed in protein lysis buffer (50 mM Tris, $150 \mathrm{mM}$ $\mathrm{NaCl}, 10 \mathrm{mM}$ EDTA, 1\% Triton X-100, $200 \mathrm{mM}$ sodium fluoride, $4 \mathrm{mM}$ sodium orthovanadate-containing protease inhibitors, $\mathrm{pH}$ 7.5) for $1 \mathrm{~h}$ on ice. Protein concentration was measured by the Bradford method. Proteins were separated by $10 \%$ sodium dodecyl sulfate-polyacrylamide electrophoresis (SDS-PAGE) and transferred to nitrocellulose membranes. Membranes were blocked with $5 \%$ bovine serum albumin (BSA) in TBST (50 mM Tris, $\mathrm{pH} 7.5,150 \mathrm{mM} \mathrm{NaCl}$, $0.05 \%$ Tween-20). The membrane was incubated at $4^{\circ} \mathrm{C}$ overnight in 5\% BSA in TBST containing one of the following primary antibodies: DRP1, 1:200; SIRT1, 1:250; Mfn1, 1:1000; Mfn2, 1:200; NRF-1, 1:500; NRF-2, 1:250; ERRß, 1:1000; or B-Actin, 1:1,000. The membrane was washed 5 times with PBST, for 5 min each wash. After washing, the membrane was incubated with horseradish peroxidase-conjugated secondary antibodies for $1 \mathrm{~h}$ at room temperature, washed with PBST, and developed with an enhanced chemiluminescence (ECL) kit (Amersham, Piscataway, NJ, USA).

ATP production. The ATP content of the adipocytes was measured with ATP lite-glo, a luciferase-based luminescence assay kit (PerkinElmer). Briefly, treated cells were mixed

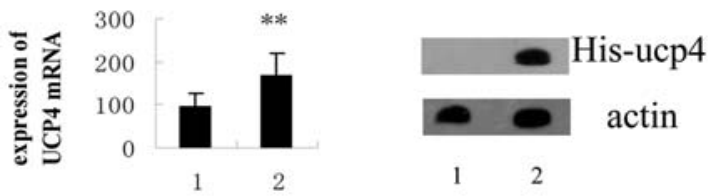

Figure 1. Expression of UCP4 in 3T3-L1 preadipocyte cells transfected with pcDNA3.1-UCP4. (A) Expression of UCP4 mRNA. Total RNA was extracted from stable lines transfected with pcDNA3.1 or pcDNA3.1-UCP4 constructs. Real-time PCR was performed using the primers indicated in Table I. Lane 1, vector (pcDNA3.1); 2, pcDNA3.1-UCP4. (B) Expression of UCP4 protein. Total proteins were isolated from stable cell lines and analyzed by Western blotting using a mouse anti-6His antibody. Lane 1, vector (pcDNA3.1); 2, pcDNA3.1-UCP4. Values represent the means \pm SD from 3 independent experiments. ${ }^{*} \mathrm{P}<0.05$ vs.vector adipocytes.

with the detection reagent for $5 \mathrm{~min}$ and luminescence was measured with a VERITAS ${ }^{\mathrm{TM}}$ Microplate Luminometer (Turner Biosystems).

$M M P$. Adipocytes were incubated with $150 \mathrm{nM}$ of Mitotracker, a red mitochondria-specific cationic fluorescent dye (Molecular Probes, Invitrogen), for $30 \mathrm{~min}$ at $37^{\circ} \mathrm{C}$, and then washed 3 times with pre-warmed phosphate-buffered saline (PBS). The spectral characteristics of the harvested cells were analyzed by fluorescence assisted cell sorting (FACS; excitation at $579 \mathrm{~nm}$, emission at $644 \mathrm{~nm}$ ). The fluorescence intensity reflects the MMP.

Measurement of intramitochondrial $\mathrm{Ca}^{2+}$. Intramitochondrial calcium $\left[\mathrm{Ca}^{2+}\right] \mathrm{m}$ in adipocytes was measured using a rhod-2 fluorescence imaging system. Mitochondrial $\mathrm{Ca}^{2+}$ levels can be monitored using anionic, membrane-permeable fluorescent molecules like Rhod-2-AM (20). This compound freely passes through the plasma membrane of the cell due to its acetoxymethyl (AM) esters, which are cleaved by intracellular esterases. Adipocytes were first washed several times with phosphatebuffered saline (PBS). Before measuring $\left[\mathrm{Ca}^{2+}\right] \mathrm{m}$, adipocytes were incubated in serum-free medium for $2 \mathrm{~h}$ and then rinsed with PBS. Adipocytes were incubated with $10 \mathrm{mM}$ rhod-2 acetoxymethyl ester (AM) (Molecular Probes, Invitrogen) in the same buffer in the dark for $1 \mathrm{~h}$ at $37^{\circ} \mathrm{C}$. The adipocytes were then rinsed three times with PBS to remove extracellular dye and washed three times with pre-warmed PBS. The spectral characteristics of harvested cells were analyzed by fluorescence assisted cell sorting (FACS; excitation at $543 \mathrm{~nm}$, emission at $615 \mathrm{~nm}$ ). The fluorescence intensity reflects the intramitochondrial $\left[\mathrm{Ca}^{2+}\right] \mathrm{m}$.

Determination of intracellular ROS content. Intracellular ROS generation was assessed using 6-carboxy-2,7-dichlorodihydrofluorescein diacetate (H2-DCFDA) as previously described (21). Cells were washed twice in KRP buffer, incubated in pre-warmed KRP containing $25 \mathrm{mM}$ glucose and $5 \mu \mathrm{M} \mathrm{H} 2-\mathrm{DCFDA}$ (Sigma), and placed at $37^{\circ} \mathrm{C}$. After $30 \mathrm{~min}$, cells were washed twice with KRP, and fluorescence was immediately measured in a plate reader using FACS (excitation at $488 \mathrm{~nm}$, emission at $525 \mathrm{~nm}$ ).

Statistical analysis. All data are expressed as means \pm SEM. Statistical analysis was performed using one-way ANOVA 
A

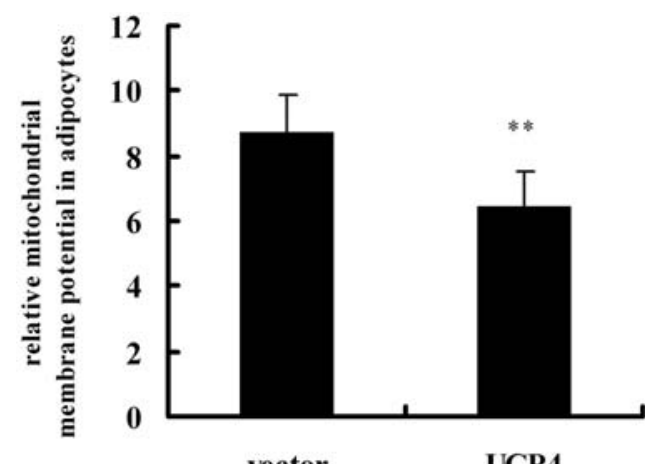

B
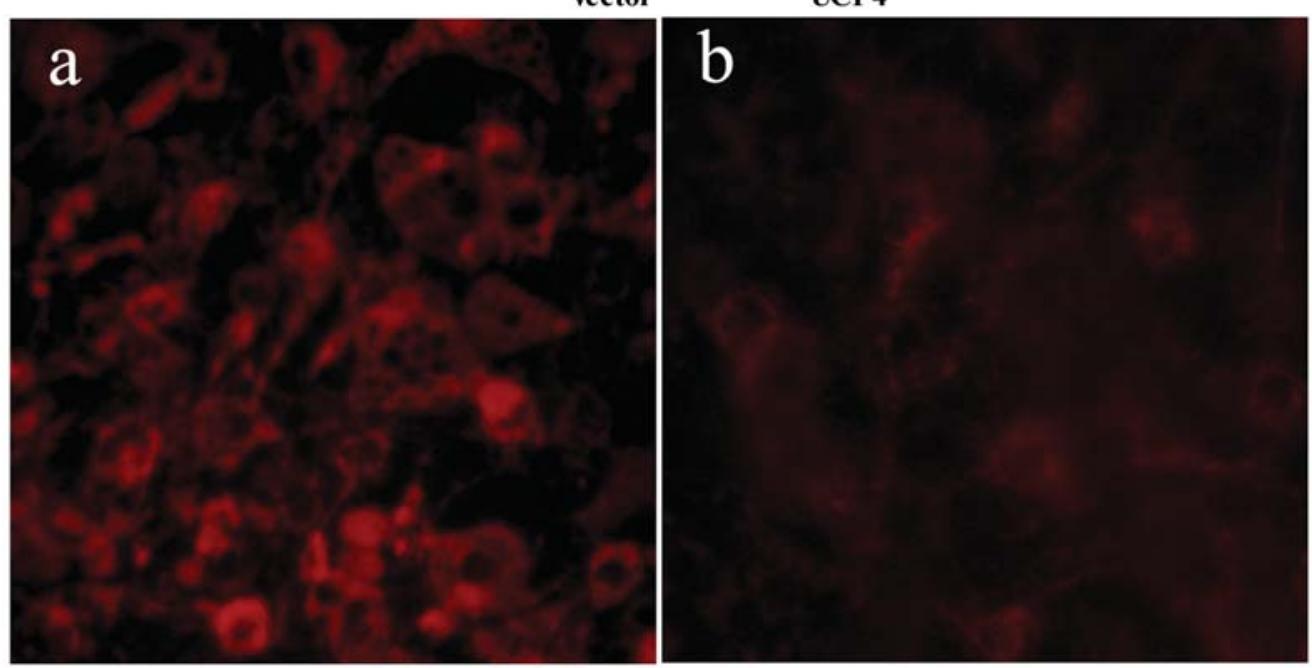

$\mathrm{C}$
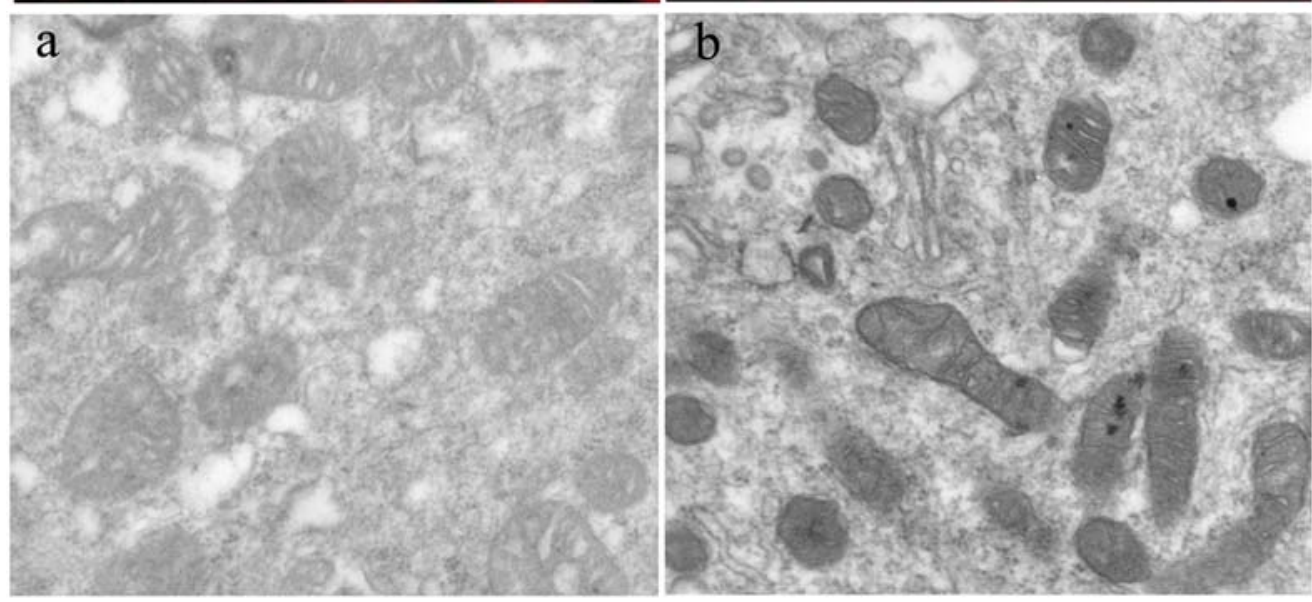

Figure 2. Mitochondrial membrane potential and mitochondria morphology are altered in adipocyte forced expression of UCP4. (A and B) Mitochondrial membrane potential. 3T3-L1 preadipocytes transfected with UCP4 or the empty vector (pcDNA3.1Myc/His B) were induced to differentiate. Mitochondrial membrane potential was displayed by mitotracker-red fluorescence probe. (A) 1 , vector; 2 , UCP4. ${ }^{*} \mathrm{P}<0.05$ vs.vector adipocytes. The mitochondrial membrane potential in adipocytes were also displayed with fluorescence microscope. a, vector; b, UCP4 (B). T-values represent the means \pm SD from 3 independent experiments. (C) The morphological features of mitochondrial 3T3-L1 preadipocytes transfected with UCP4 or the empty vector (pcDNA3.1Myc/His B) were induced to differentiate. Ultra-structural analysis of mitochondrial adipocytes were visualized by transmission electron microscopy. a, vector; b UCP4, (x 50,000).

with the SPSS 10.0 statistical software package (SPSS Inc., Chicago, IL, USA). The threshold of significance was defined as $\mathrm{P}<0.05$.

\section{Results}

Stable expression of UCP4. A 3T3-L1 cell line stably expressing a transfected UCP4 plasmid was established and maintained in DMEM containing $200 \mu \mathrm{g} / \mathrm{ml}$ G418. Expression of UCP4 was verified by real-time PCR (Fig. 1A) and Western blotting (Fig. 1B).
MMP and mitochondrial morphology are altered in adipocytes overexpressing UCP4. UCPs are located on the mitochondrial inner membrane and function as proton transporters. To determine whether UCP4 overexpression has an effect on mitochondria, we monitored MMP and assessed the ultrastructure of mitochondria in adipocytes after the stable transfection of UCP4. We found that UCP4 overexpression resulted in a drop in MMP (Fig. 2A), as shown by the potential dependent fluorescence probe. Additionally, UCP4-forced cells displayed condensed mitochondria with twisted and condensed cristae (Fig. 2B). The vector adipocytes, however, 
showed clear and oriented cristae that were normal in shape. The abnormal MMP and the morphology of these mitochondria motivated us to track other mitochondria related events.

Mitochondrial fusion and fission are impaired and mitobiogenesis is reduced upon overexpression of UCP4. To further clarify the mechanisms underlying the effects of UCP4 on mitochondrial morphology, we examined the levels of the mitochondrial fusion and fission proteins $\mathrm{mfn} 1, \mathrm{mfn} 2$, and Drp1 (Fig. 3A). These proteins mediate the main fusion and fission processes in mammals, and ultimately determine the shape of the mitochondria. Compared to control vector adipocytes, $\mathrm{mfn} 1$ protein levels were considerably reduced in adipocytes upon UCP4 expression, while a slight diminishment of mfn2 was observed. Mfn1 and Mfn2 share $>70 \%$ sequence similarity (22). In the present study, levels of $\mathrm{mfn} 1$ were markedly altered, indicating that it plays a more central role in mitochondrial deformation. Levels of the fission protein Drp1 were strikingly decreased upon UCP4 expression. Altogether, the mitochondria size displayed was the same as vector adipocytes, which may be attributed to the simultaneous impairment of mitofusin and mitofission.

We also examined the expression levels of the mitochondrial biogenesis-related factors PGC- $1 \alpha$ and PGC-1ß. The expression of PGC-1 $\alpha$ mRNA (Fig. 3B) was drastically decreased after UCP4 overexpression. The level of PGC-1ß protein maintained no alteration. PGC-1ß protein located between 39 and $51 \mathrm{kDa}$ with double presence, this was consistent with the antibody directions provided by the manufacturer. To further explore the changes in mitochondrial biogenesis, we also assessed the levels of NRF-1, NRF-2, ERRß, SIRT1, and mtTFA. NRF-1 and ERRß protein levels were greatly decreased after UCP4 expression. ERRß protein located between 38 and $59 \mathrm{kDa}$ with double presence, this was consistent with the antibody directions provided by the manufacturer. However, levels of NRF-2 were dramatically elevated after UCP4 expression. Levels of SIRT1, which deacetylates and then activates PGC-1 $\alpha$, were the same in both vector and UCP4 adipocytes. mRNA expression of mtTFA (Fig. 3B), a key factor in mitochondrial biogenesis, was reduced upon UCP4 expression. These data indicate that mitochondrial biogenesis is greatly altered by UCP4 overexpression.

mtDNA and ROS production are affected by UCP4 expression. Since mitochondrial biogenesis was drastically affected by UCP4 overexpression, we next investigated whether UCP4 overexpression affected mtDNA copy number and ROS production (Fig. 4A). By real-time PCR, we revealed that mtDNA levels decreased significantly upon UCP4 overexpression. We know that mtDNA only encodes for two ribosomal RNAs, the 22 transfer RNA and the 13 messenger RNA, which are exclusively involved in the translation of the subunits of complexes I, III, IV and V of the mitochondrial respiratory chain (MRC). Since the activity of the MRC is closely related to ROS production, we next sought to determine whether UCP4 overexpression affected ROS production. ROS levels in UCP4 overexpressing adipocytes were strikingly enhanced, as indicated by greater fluorescence in the presence of the compound DFCDA (Fig. 4B and C).

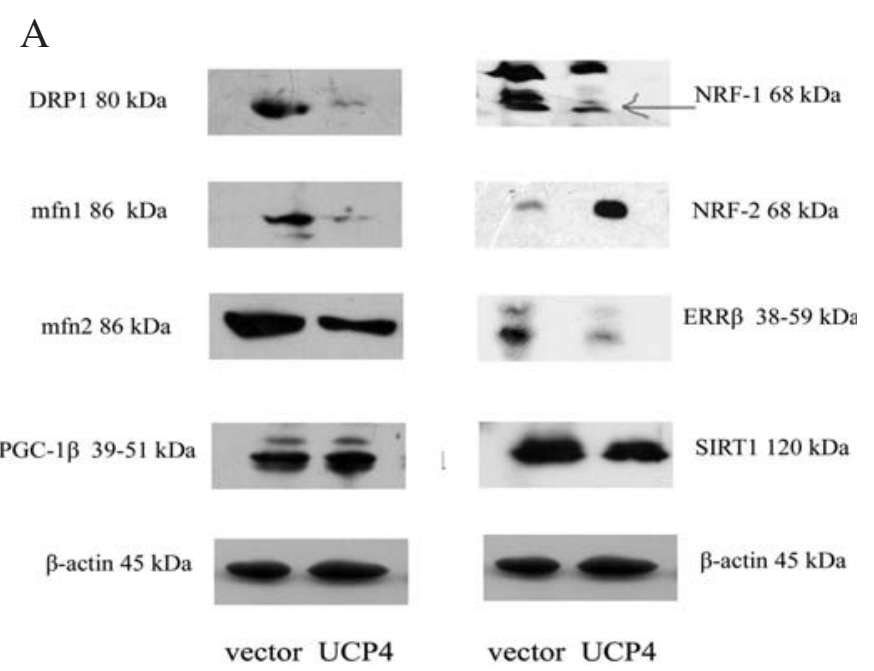

B

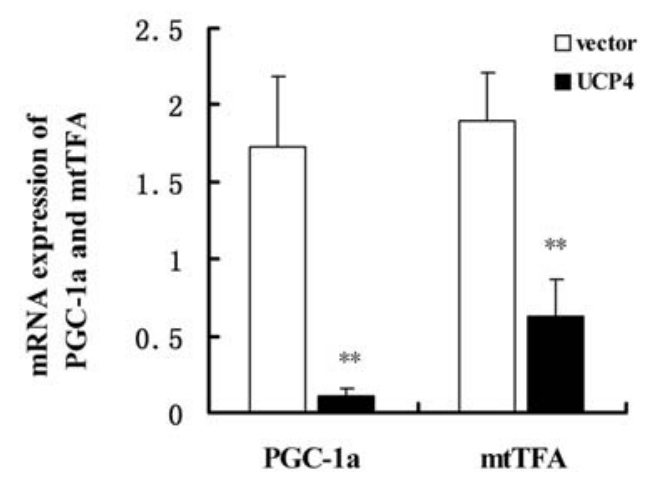

Figure 3. Effects of UCP4 on mitochondrial biogenesis. 3T3-L1 preadipocytes transfected with UCP4 or the empty vector (pcDNA3.1Myc/His B) were induced to differentiate. Cell lysates were analyzed by SDS-PAGE, blotted onto a membrane, and then probed with corresponding antibodies, $\beta$-actin as an internal control (A). The RNA was extracted and relative expression of PGC- $1 \alpha$ and mtTFA mRNA was examined by real-time RT-PCR (B). B-actin mRNA as internal control. Values are shown as mean $\pm \mathrm{SD}$ of triplicate; 1 , vector; $2, \mathrm{UCP} 4 ;{ }^{* *} \mathrm{P}<0.01$ vs. vector adipocytes.

Intracellular ATP and intramitochondrial $\mathrm{Ca}^{2+}$ concentration are decreased in adipocytes upon overexpression of UCP4. It is well known that uncoupling proteins dissipate the mitochondrial proton gradient, thus uncoupling oxidative phosphorylation and allowing stored energy to be expended as heat. We next assessed intracellular ATP concentration (Fig. 5A). As we expected, the intracellular ATP concentration was decreased upon UCP4 overexpression. UCP4 overexpression led to a large reduction in intramitochondrial calcium concentration in mature adipocytes (Fig. 5B and C). These results clearly demonstrate that UCP4 overexpression induces mitochondrial dysfunction in adipocytes.

Insulin sensitivity was impaired upon UCP4 overexpression. An association between mitochondrial dysfunction and reduced insulin sensitivity has been observed in cross-sectional studies (23). We next investigated the insulin sensitivity after UCP4 forced expression. As shown in Fig. 6, insulin stimulated 2-Deoxy-D-[3H] glucose uptake was greatly lessened when compared with vector adipocytes. In other words, the adipocytes became insulin resistant after UCP4 overexpression. 
A

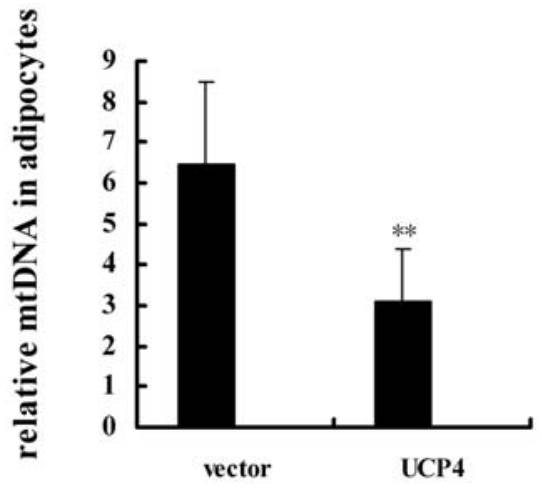

$\mathrm{C}$

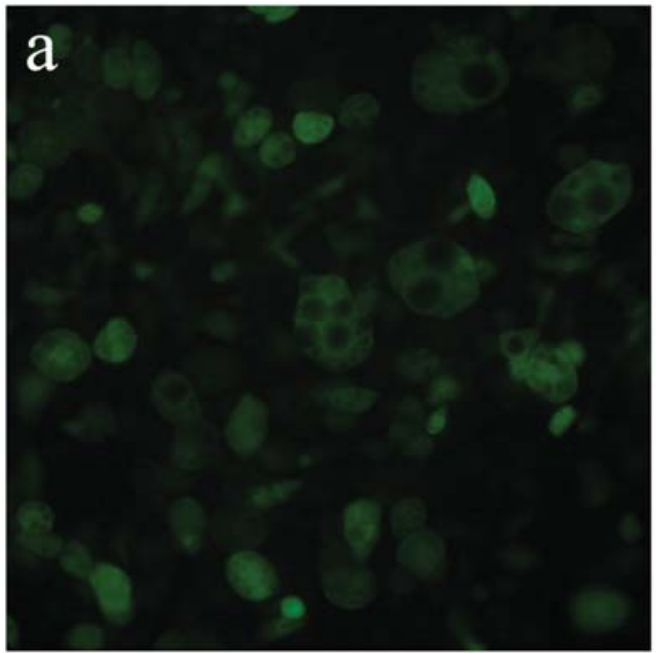

B

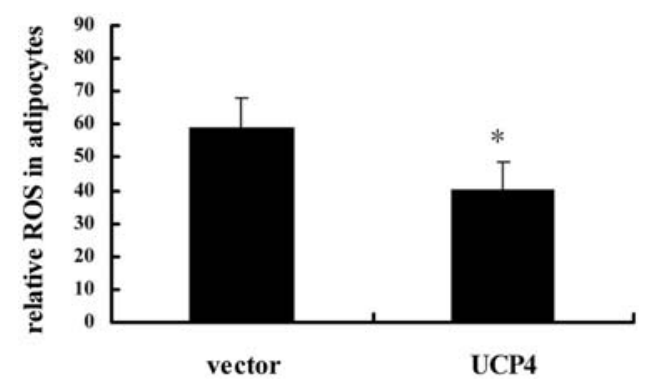

Figure 4. mtDNA and ROS content are affected by UCP4 expression. 3T3-L1 preadipocytes transfected with UCP4 or the empty vector (pcDNA3.1Myc/His B) were induced to differentiate. Total DNA was extracted and relative expression levels of cytB copynumber were determined by real-time RT-PCR and normalized with 28s (A). Total cellular ROS levels were measured with DCF fluorescence probe by FCM (B) and the ROS in adipocytes were displayed with a fluorescence microscope (C). a, vector; b, UCP4. Values represent the means \pm SD from 3 independent experiments. ${ }^{* *} \mathrm{P}<0.01$ vs.vector adipocytes.

\section{Discussion}

The major findings of our study are as follows. i) UCP4 overexpression resulted in mitochondrial deformation and reduced MMP. Mitochondria in UCP4 expressing adipocytes displayed compact and unclear cristae; ii) Mitochondrial fusion and fission became imbalanced and mitobiogenesis was reduced upon overexpression of UCP4. Mitochondrial biogenesis factors (NRF-1, NRF-2, PGC-1 $\alpha$, mtTFA, and ERRß) and mitochondrial mtDNA were all changed; iii) Intracellular ROS levels increased and ATP levels were reduced, which was accompanied by an attenuated intramitochdrial calcium level; iv) We found that adipocytes became insensitive to insulin. Apparently, UCP4 expression in 3T3-L1 has an impact on mitochondrial function.

Mitochondrial morphology is closely related to mitochondrial function and metabolic activity (24). Several reports have described an association between mitochondrial dysfunction and alterations in mitochondrial morphology $(25,26)$. The mitochondrial matrix and cristae are the main sites for metabolism, and condensed mitochondria do not contain enough space to maintain normal or super metabolic needs. Moreover, when UCP4 was overexpressed there was reduced MMP and functional mitochondria were fewer, which is in line with previously reported data (13). UCPs have the basal function of uncoupling the electrochemical proton gradient and oxidative phosphorylation (OxPhos). UCP4 overexpression resulted in increased proton leakage, which depolarized the MMP.

To discover the cause for condensed mitochondria, we investigated the mitochondrial fusion and fission related proteins. The balance between fusion and fission plays a central role in controlling mitochondrial morphology (27). Mfn1 and Mfn2 are mitochondrial GTPases required for mitochondrial fusion. Mfn1 tethers to mitochondrial membranes more efficiently than Mfn2 (28). The disruption of mitochondrial fusion by knockdown of Mfns leads to mitochondrial fragmentation (29). While DRP1 mediates mitochondrial fission, preventing mitochondrial fission by downregulating expression of Drp1 leads to mitochondrial dysfunction, an increase in cellular ROS, and a loss of mtDNA, which correlates with a depletion of cellular ATP, inhibition of cell proliferation, and autophagy (30). Mitochondria frequently undergo fusion and fission so that they can effectively exchange power and content. UCP4 overexpression downregulated $\mathrm{mfn} 1, \mathrm{mfn} 2$, and DRP1, leading to decreased mitochondrial fusion and fission, which meant exchange activity slowed down. In addition, inhibition of mitochondrial fusion has been shown to reduce the activity of the electron transfer chain (ETC) (31), and to reduce mitochondrial metabolism (32).

Mitochondrial fusion and fission also determined mitochondrial numbers and biogenesis. MtDNA and key factors 
A

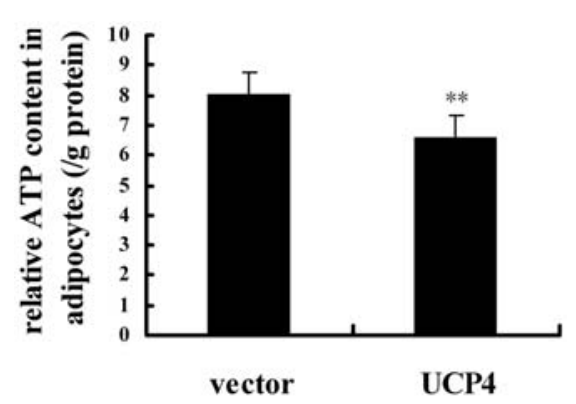

$\mathrm{C}$

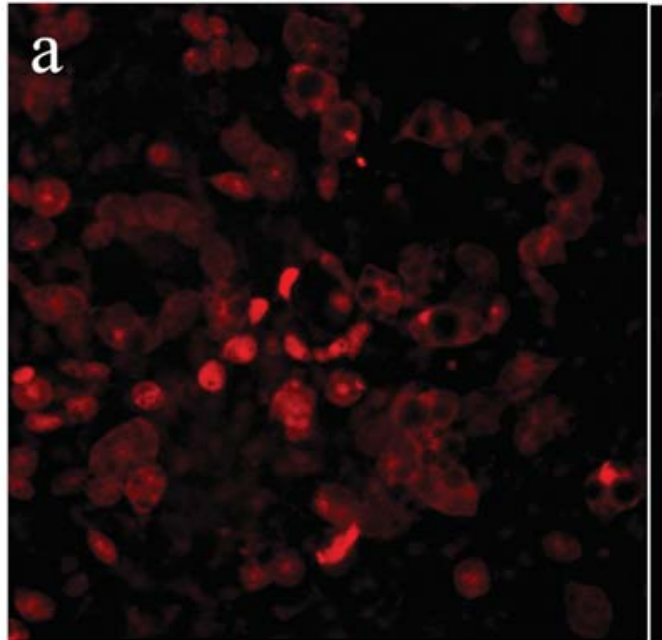

B

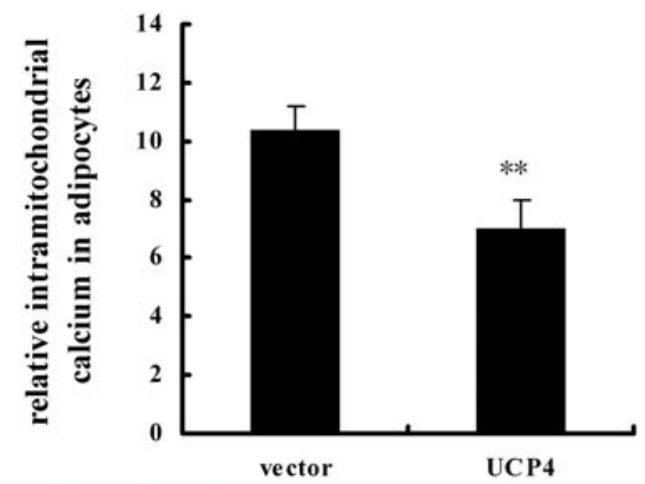

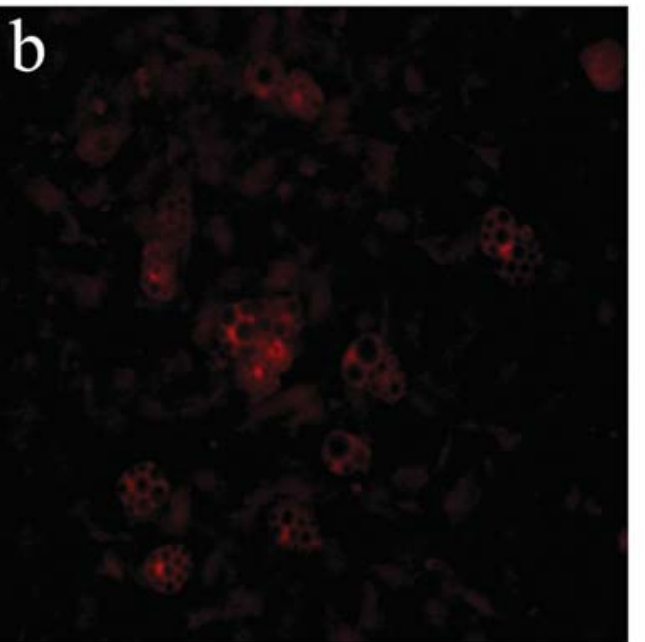

Figure 5. Intracellular ATP and intramitochondrial calcium concentration are decreased in adipocytes upon forced expression of UCP4. 3T3-L1 preadipocytes transfected with UCP4 or the empty vector (pcDNA3.1Myc/His B) were induced to differentiate. Intracellular ATP was determined by ATPlite-glo, a luciferase-based luminescence assay kit, according to the manufacturer's direction (A). 1, vector; 2, UCP4. Intramitochondrial calcium levels were performed by rhod-2 fluoresence probe by FCM (B). The intramitochondrial calcium in adipocytes were also displayed with fluorescence microscope (C), a, vector; b, UCP4. Values represent the means \pm SD from 3 independent experiments. ${ }^{* *} \mathrm{P}<0.01$ vs.vector adipocytes.

of mitochondrial biogenesis, PGC-1 $\alpha$ and mtTFA, were reduced by UCP4 overexpression. We also observed reduced levels of NRF-1 protein and mtTFA mRNA, both targets of PGC-1. $\mathrm{NRF}-1$ is a transcription factor that stimulates the transcription of many nuclear-encoded mitochondrial genes, such as OXPHOS genes and mtTFA. mtTFA can bind to the D-loop of the mitochondrial genome and promote transcription of mitochondrial genes and replication of mitochondrial DNA (33). However, levels of SIRT1, which deacetylates and then activates PGC- $1 \alpha$, were the same in UCP4 and vector adipocytes. We saw no change in another key biogenesis factor, PGC-1ß. An interesting result was that NRF-2 increased upon UCP4 expression. NRF-1 and NRF-2 have been implicated in the expression of many respiratory subunits along with key components of nuclear-encoded mitochondrial gene transcription, replication and biosynthetic machinery (34). Mutation of the NRF-1-binding site abolished Tfam activation, but mutation of NRF-2 only slightly affected its activation (35). In the absence of a certain level of NRF-1, NRF-2 plays only a marginal role in Tfam in vivo expression (36). Our results provide further evidence that while NRF-2 level increased greatly, TFAM mRNA was kept at a low level. ERRß is an orphan receptor that is reported to exhibit constitutive transcriptional activity. In addition, its function is also regulated by PGC- $1 \alpha$ and PGC-1ß $(34,37)$. Targeted disruption of the ERRß gene in mice resulted in severely impaired placental

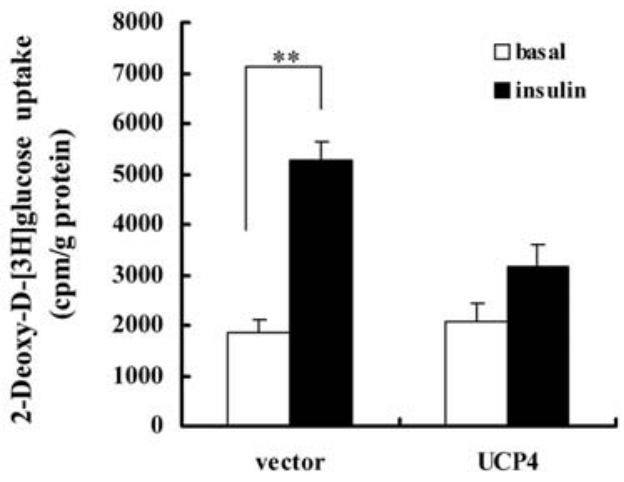

Figure 6. Effect of UCP4 on glucose uptake. 3T3-L1 preadipocytes transfected with UCP4 or the empty vector (pcDNA3.1Myc/His B) were induced to differentiate. After serum starvation for $3 \mathrm{~h}$, cells were incubated with (black columns) or without (white columns) $100 \mathrm{nmol} / 1$ insulin for $30 \mathrm{~min}$, followed by measurement of the 2-deoxy-D-[3H] glucose uptake. Values represent the means $\pm \mathrm{SD}$ from 3 independent experiments. ${ }^{* *} \mathrm{P}=0.001<0.01$ vs. insulin-stimulated control (cells transfected with the empty vector).

formation, and the embryo died at 10.5 days post-coitum (38). ERRß overexpressed prostate cancer cell lines displayed more active mitochondria at high membrane potentials and showed improved lipid metabolism (39). Recent work has established that ERRs play a role in regulating energy metabolism and mitochondrial biogenesis and function $(37,40)$. Our results 
indicated that UCP4 had a profound effect on mitochondrial energy metabolism and biogenesis, and led to mitochondrial dysfunction.

Previously, Hackenbrock has shown that isolated rat-liver mitochondria switch from a condensed to an orthodox state when [ADP] drops during the conversion from respiratory state 3 to 4 (41). Mitochondria in intact cells respire between the extreme energetic states, state 3 (in the presence of ADP) and state 4 (when ADP has been converted into ATP), as described by studies on isolated mitochondria (42). Condensed mitochondria indicate that ATP production had decreased and this was shown in our study. Our results indicated that UCP4 overexpressing adipocytes displayed condensed mitochondria and reduced ATP production. This structural transition coincides with increased intracellular production of ROS. It was reported that mild uncoupling decreased ROS production. Whereas overexpression of UCP4 (perhaps too much uncoupling) induced a series of metabolic disorders, including sequential mitochondrial dysfunction and ROS accumulation, which did not compensate for the effects of decreasing ROS production. We also observed that intramitochondrial calcium was lost in UCP4 expressing adipocytes. The mitochondria are a major $\mathrm{Ca}^{2+}$ storage organelle, and can take up $\mathrm{Ca}^{2+}$ from the endoplasmic reticulum (ER). Mitochondrial $\mathrm{Ca}^{2+}$ regulation is achieved primarily via the mitochondrial $\mathrm{Ca}^{2+}$ uniporters, whereby $\mathrm{Ca}^{2+}$ is taken up by means of a potential difference. Carbonyl cyanide p-trifluoromethoxyphenylhydrazone (FCCP)-induced mitochondrial uncoupling causes depolarization and $\mathrm{Ca}^{2+}$ release from the ER and mitochondria in brown adipocytes (43). This was shown in our investigation, since UCP4 overexpression rendered deplorization and intramitochondrial calcium was lost. Moreover, studies on cultured mammalian cells have shown that the formation of a reticular mitochondrial network is important for proper mitochondrial calcium buffering and for propagating intramitochondrial $\mathrm{Ca}^{2+}$ waves $(44,45)$. The interaction between ROS and calcium has also been extensively investigated (46-48). Calcium signaling is essential for the production of ROS, and elevated intracellular calcium $\left(\left[\mathrm{Ca}^{2+}\right] \mathrm{i}\right)$ activates ROS-generating enzymes, such as NADPH oxidase and myeloperoxidase, as well as inducing the formation of free radicals by the mitochondrial respiratory chain (49). Taken together, mitochondrial deformation and intramitochondrial calcium, as well as ROS production, are closely related to $\mathrm{UCP} 4$ overexpression and the loss of MMP.

Finally, overexpression of UCP4 resulted in adipocyte insulin resistance, indicated by decreased insulin stimulated glucose uptake. This coincided with the abnormal mitochondria seen in our results. A growing body of evidence has linked mitochondrial dysfunction to insulin resistance and type 2 diabetes $(50,51)$. Deformed mitochondria and elevated ROS maybe the contributors to insulin resistance, and mitochondrial dysfunction has emerged in UCP4 expressing adipocytes, as indicated by abnormal mitochondrial biogenesis and decreased ATP production. However, in other cells, UCP4 overexpression plays a protective role (16). There are five UCPs being widely investigated, all of which had been shown to be antioxidants and cell protectors. For example, ectopic UCP1 expression stimulated mitochondrial biogenesis in unilocular adipocytes in vivo (52), and UCP2 may provide cytoprotection upon hypoxic stress (53) or neuroprotection by inducing mild mitochondrial uncoupling, which prevents mitochondrial release of pro-apoptotic proteins (54). Overexpression of UCP3 prevents glucose-induced transient mitochondrial membrane hyperpolarization, ROS formation, and induction of PCD (55). Overexpression of UCP3 in skeletal muscle protects against fat-induced insulin resistance (56). Knockdown of UCP5 expression affected MMP after $\mathrm{MPP}^{+}$exposure and induced apoptosis (57).

Whereas, UCP2 expression was shown to be a factor leading to necrosis, UCP2-mediated reduction in mitochondrial function contributes to mitochondrial dysfunction and the necrotic death of primary cultured mesencephalic cells (MCs) after exposure to cyanide, a complex IV inhibitor (58). The targeted suppression of UCP2 expression in fatty liver cells can alleviate the ischemia/reperfusion injury in ob/ob mice (59). Overexpression of UCP2 in these cells switched the response to necrosis, which was related to a greater level of mitochondrial dysfunction. Similar to these results, UCP4 overexpression in adipocytes induced mitochondrial dysfunction and ROS production, which could be explained by the moderate to strong uncoupling, which markedly reduced ATP levels in cells and induced non-apoptotic cell death (60). Like UCP2, induction of UCP4 expression may be a double-edged sword; increased action would reduce the efficiency of ATP synthesis and reduce ROS generation to decrease the execution of apoptosis (61).

We previously showed that overexpression of UCP4 can inhibit the apoptosis of preadipocytes (17). Although we have not found evidence for necrosis under TEM, the mitochondrial MMP, loss of intramitochondrial calcium, and decreased ATP levels suggested a disorder induced by UCP4 overexpression. We considered that, i) in our study we used mature adipocytes, while previous studies used preadipocytes. Preadipocytes, which are fibroblasts, did not represent the metabolic state of mature adipocytes; ii) The UCP4 induced dysfunction had not reached the degree of necrosis and iii) UCP4 may have different impacts on apoptosis and necrosis execution programs. Overexpression of UCP2 in HeLa cells decreased MMP, NADH, and ATP levels, and subsequent cell death was not prevented by caspase inhibitors (60). Although UCP4 expression inhibited the apoptosis of preadipocytes, we could not conclude whether UCP4 played a role in mature adipocyte apoptosis.

In conclusion, the results of this study demonstrate that UCP4 overexpression inhibits insulin-stimulated glucose uptake in mature adipocytes and induces mitochondrial dysfunction. These disorders, induced by UCP4 overexpression, provide clues for comprehending mitochondrial dysfunction and insulin resistance.

\section{Acknowledgements}

We gratefully acknowledge Dr Yu-hui Ni for expert technical assistance. This work was supported by grants from the National Natural Science Foundation of China (Grant Number: 30772364), the Foundation of Ministry of Education, China (Grant Number: 20070312001), the Natural Science Foundation of Jiangsu Province, China (Grant Number: BK2007230), and Nanjing Medical University (Grant Number: 07NMUZ029). 


\section{References}

1. McGarry JD: Banting lecture 2001: dysregulation of fatty acid metabolism in the etiology of type 2 diabetes. Diabetes 51: 7-18, 2002.

2. Bonnard C, Durand A, Peyrol S, Chanseaume E, Chauvin MA, Morio B, Vidal H and Rieusset J: Mitochondrial dysfunction results from oxidative stress in the skeletal muscle of dietinduced insulin-resistant mice. J Clin Invest 118: 789-800, 2008.

3. Newsholme P, Haber EP, Hirabara SM, Rebelato EL, Procopio J, Morgan D, Oliveira-Emilio HC, Carpinelli AR and Curi R: Diabetes associated cell stress and dysfunction: role of mitochondrial and non-mitochondrial ROS production and activity. J Physiol 583: 9-24, 2007

4. Abdul-Ghani MA, Jani R, Chavez A, Molina-Carrion M, Tripathy D and Defronzo RA: Mitochondrial reactive oxygen species generation in obese non-diabetic and type 2 diabetic participants. Diabetologia 52: 574-582, 2009.

5. Houstis N, Rosen ED and Lander ES: Reactive oxygen species have a causal role in multiple forms of insulin resistance. Nature 440: 944-948, 2006.

6. Kim JK, Fillmore JJ, Sunshine MJ, Albrecht B, Higashimori T, Kim DW, Liu ZX, Soos TJ, Cline GW, O'Brien WR, Littman DR and Shulman GI: PKC-theta knockout mice are protected from fat-induced insulin resistance. J Clin Invest 114: 823-827, 2004.

7. Yu CL, Chen Y, Cline GW, Zhang DY, Zong HH, Wang YL, Bergeron R, Kim JK, Cushman SW, Cooney GJ, Atcheson B, White MF, Kraegen EW and Shulman GI: Mechanism by which fatty acids inhibit insulin activation of insulin receptor substrate-1 (IRS-1)-associated phosphatidylinositol 3-kinase activity in muscle. J Biol Chem 277: 50230-50236, 2002.

8. Um SH, Frigerio F, Watanabe M, Picard F, Joaquin M, Sticker M, Fumagalli S, Allegrini PR, Kozma SC, Auwerx J and Thomas G: Absence of S6K1 protects against age- and diet-induced obesity while enhancing insulin sensitivity. Nature 431: 200-205, 2004.

9. Davis JE, Gabler NK, Walker-Daniels J and Spurlock ME: The c-Jun N-terminal kinase mediates the induction of oxidative stress and insulin resistance by palmitate and toll-like receptor 2 and 4 ligands in 3T3-L1 adipocytes. Horm Metab Res 41: 523-530, 2009.

10. Taniguchi CM, Aleman JO, Ueki K, Luo J, Asano T, Kaneto H, Stephanopoulos G, Cantley Lc and Kahn CR: The p85alpha regulatory subunit of phosphoinositide 3-kinase potentiates C-Jun $\mathrm{N}$-terminal kinase-mediated insulin resistance. Mol Cell Biol 27: 2830-2840, 2007

11. Hirosumi J, Tuncman G, Chang LF, Gorgun CZ, Uysal KT, Maeda K, Karin M and Hotamisligil GS: A central role for JNK in obesity and insulin resistance. Nature 420: 333-336, 2002

12. Daval M, Foufelle F and Ferré P: Functions of AMP-activated protein kinase in adipose tissue. J Physiol 574: 55-62, 2006.

13. Mao W, Yu XX, Zhong A, Li W, Brush J, Sherwood SW Adams SH and Pan G: UCP4, a novel brain-specific mitochondrial protein that reduces membrane potential in mammalian cells FEBS Lett 443: 326-330, 1999.

14. Chan SL, Liu D, Kyriazis GA, Bagsiyao P, Ouyang X and Mattson MP: mitochondrial uncoupling protein-4 regulates calcium homeostasis and sensitivity to store depletion-induced apoptosis in neural cells. J Biol Chem 281: 37391-37403, 2006.

15. Guevara R, Santandreu FM, Valle A, Gianotti M, Oliver J and Roca P: Sex-dependent differences in aged rat brain mitochondrial function and oxidative stress. Free Radic Biol Med 46: 169-175, 2009.

16. Chu AC, Ho PW, Kwok KH, Ho JW, Chan KH, Liu HF, Kung MH, Ramsden DB and Ho SL: Mitochondrial UCP4 attenuates $\mathrm{MPP}^{+}$and dopamine-induced oxidative stress, mitochondrial depolarization, and ATP deficiency in neurons and is interlinked with UCP2 expression. Free Radic Biol Med 46: 810-820, 2009

17. Zhang M, Wang B, Ni YH, Liu F, Fei L, Pan XQ, Guo M, Chen RH and Guo XR: Overexpression of uncoupling protein 4 promotes proliferation and inhibits apoptosis and differentiation of preadipocytes. Life Sci 79: 1428-1435, 2006.

18. Ceddia RB, Somwar R, Maida A, Fang X, Bikopoulos G and Sweeney G: Globular adiponectin increases GLUT4 translocation and glucose uptake but reduces glycogen synthesis in rat skeletal muscle cells. Diabetologia 48: 132-139, 2005.

19. He L, Chinnery PF, Durham SE, Blakely EL, Wardell TM, Borthwick GM, Taylor RW and Turnbull DM: Detection and quantification of mitochondrial DNA deletions in individual cells by real-time PCR. Nucleic Acids Res 30: E68, 2002.
20. Visch HJ, Koopman WJ, Zeegers D, van Emst-de Vries SE, van Kuppeveld FJ, van den Heuvel LW, Smeitink JA and Willems PH: $\mathrm{Ca}^{2+}$-mobilizing agonists increase mitochondrial ATP production to accelerate cytosolic $\mathrm{Ca}^{2+}$ removal: aberrations in human complex I deficiency. Am J Physiol Cell Physiol 291: C308-C316, 2006.

21. Park J, Choe SS, Choi AH, Kim KH, Yoon M J, Suganami T, Ogawa Y and Kim JB: Increase in glucose-6-phosphate dehydrogenase in adipocytes stimulates oxidative stress and inflammatory signals. Diabetes 55: 2939-2949, 2006.

22. Chen H, Detmer SA, Ewald AJ, Griffin EE, Fraser SE and Chan DC: Mitofusins Mfn1 and Mfn2 coordinately regulate mitochondrial fusion and are essential for embryonic development. J Cell Biol 160: 189-200, 2003.

23. Sirikul B, Gower BA, Hunter GR, Larson-Meyer DE and Newcomer BR: Relationship between insulin sensitivity and in vivo mitochondrial function in skeletal muscle. Am J Physiol Endocrinol Metab 291: E724-E728, 2006.

24. Mannella CA: Structure and dynamics of the mitochondrial inner membrane cristae. Biochim Biophys Acta 1763: 542-548, 2006.

25. Katakam PV, Jordan JE, Snipes JA, Tulbert CD, Miller AW and Busija DW: Myocardial preconditioning against ischemiareperfusion injury is abolished in Zucker obese rats with insulin resistance. Am J Physiol Regul Integr Comp Physiol 292: R920-R926, 2007.

26. Yu T, Robotham JL and Yoon Y: Increased production of reactive oxygen species in hyperglycemic conditions requires dynamic change of mitochondrial morphology. Proc Natl Acad Sci USA 103: 2653-2658, 2006.

27. Santel A: Get the balance right: mitofusins roles in health and disease. Biochim Biophys Acta 1763: 490-499, 2006.

28. Ishihara N, Eura Y and Mihara K: Mitofusin 1 and 2 play distinct roles in mitochondrial fusion reactions via GTPase activity. J Cell Sci 117: 6535-6546, 2004.

29. De Brito OM and Scorrano L: Mitofusin 2 tethers endoplasmic reticulum to mitochondria. Nature 456: 605-612, 2008.

30. Parone PA, Da Cruz S, Tondera D, Mattenberger Y, James DI, Maechler P, Barja F and Martinou JC: Preventing mitochondrial fission impairs mitochondrial function and leads to loss of mitochondrial DNA. PLoS ONE 3: E3257, 2008

31. Masako K, Yoko H, Hisashi S, Ryotaro H and Kenji K: Bidirectional $\mathrm{Ca}^{2+}$ coupling of mitochondria with the endoplasmic reticulum and regulation of multimodal $\mathrm{Ca}^{2+}$ entries in rat brown adipocytes. Am J Physiol Cell Physiol 292: C896-C908, 2007.

32. Chen H, Chomyn A and Chan DC: Disruption of fusion results in mitochondrial heterogeneity and dysfunction. J Biol Chem 280: 26185-26192, 2005

33. Savagner F, Mirebeau D, Jacques C, Guyetant S, Morgan C, Franc B, Reynier P and Malthièry Y: PGC-1-related coactivator and targets are upregulated in thyroid oncocytoma. Biochem Biophys Res Commun 310: 779-784, 2003.

34. Kamei Y, Ohizumi H, Fujitani Y, Nemoto T, Tanaka T, Takahashi N, Kawada T, Miyoshi M, Ezaki O and Kakizuka A: PPARgamma coactivator 1beta/ERR ligand 1 is an ERR protein ligand, whose expression induces a high-energy expenditure and antagonizes obesity. Proc Natl Acad Sci USA 100: 12378-12383, 2003.

35. Wu Z, Puigserver P, Andersson U, Zhang C, Adelmant G Mootha V, Troy A, Cinti S, Lowell B, Scarpulla RC and Spiegelman BM: Mechanisms controlling mitochondrial biogenesis and respiration through the thermogenic coactivator PGC-1. Cell 98: 115-124, 1999.

36. Rodríguez-Peña A, Escrivá $\mathrm{H}$, Handler AC and Vallejo CG: Thyroid hormone increases transcription of GA-binding protein/nuclear respiratory factor-2 alpha-subunit in rat liver. FEBS Lett 514: 309-314, 2002.

37. Puigserver P and Spiegelman BM: Peroxisome proliferatoractivated receptorgamma coactivator 1 alpha (PGC-1 alpha): transcriptional coactivator and metabolic regulator. Endocr Rev 24: 78-90, 2003

38. Luo J, Sladek R, Bader JA, Matthyssen A, Rossant J and Giguère V: Placental abnormalities in mouse embryos lacking the orphan nuclear receptor ERR-beta. Nature 388: 778-782, 1997.

39. Yu S, Wong YC, Wang XH, Ling MT, Ng CF, Chen S and Chan FL: Orphan nuclear receptor estrogen-related receptorbeta suppresses in vitro and in vivo growth of prostate cancer cells via p21(WAF1/CIP1) induction and as a potential therapeutic target in prostate cancer. Oncogene 27: 3313-3328, 2008.

40. Giguère V: Transcriptional control of energy homeostasis by the estrogen-related receptors. Endocr Rev 29: 677-696, 2008. 
41. Hackenbrock CR: Ultrastructural bases for metabolically linked mechanical activity in mitochondria. I. Reversible ultrastructural changes with change in metabolic steady state in isolated liver mitochondria. J Cell Biol 30: 269-297, 1966.

42. Hüttemann M, Lee I, Pecinova A, Pecina P, Przyklenk K and Doan JW: Regulation of oxidative phosphorylation, the mitochondrial membrane potential, and their role in human disease. J Bioenerg Biomembr 40: 445-456, 2008.

43. Frieden M, James D, Castelbou C, Danckaert A, Martinou JC and Demaurex $\mathrm{N}$ : $\mathrm{Ca}(2+)$ homeostasis during mitochondrial fragmentation and perinuclear clustering induced by hFis 1 . J Biol Chem 279: 22704-22714, 2004.

44. Rizzuto R and Pozzan T: Microdomains of intracellular $\mathrm{Ca}^{2+}$ : molecular determinants and functional consequences. Physiol Rev 6: 369-408, 2006.

45. Szabadkai G, Simoni AM, Chami M, Wieckowski MR, Youle RJ and Rizzuto R: Drp-1-dependent division of the mitochondrial network blocks intraorganellar $\mathrm{Ca}^{2+}$ waves and protects against $\mathrm{Ca}^{2+}$-mediated apoptosis. Mol Cell 16: 59-68, 2004

46. Pich S, Bach D, Briones P, Liesa M, Camps M, Testar X, Palacín M and Zorzano A: The Charcot-Marie-Tooth type 2A gene product, Mfn2, up-regulates fuel oxidation through expression of OXPHOS system. Hum Mol Genet 14 : 1405-1415, 2005.

47. Toescu EC: Hypoxia sensing and pathways of cytosolic $\mathrm{Ca}^{2+}$ increases. Cell Calcium 36: 187-199, 2004.

48. Brookes PS: Mitochondrial $\mathrm{H}(+)$ leak and ROS generation: an odd couple. Free Radic Biol Med 38: 12-23, 2005.

49. Miwa S and Brand MD: Mitochondrial matrix reactive oxygen species production is very sensitive to mild uncoupling. Biochem Soc Trans 31: 1300-1301, 2003.

50. Choo HJ, Kim JH, Kwon OB, Lee CS, Mun JY, Han SS, Yoon YS Yoon G, Choi KM and Ko YG: Mitochondria are impaired in the adipocytes of type 2 diabetic mice. Diabetologia 49: 784-791, 2006.

51. Duchen MR: Roles of mitochondria in health and disease. Diabetes 53: S96-S102, 2004.

52. Rossmeisl M, Barbatelli G, Flachs P, Brauner P, Zingaretti MC, Marelli M, Janovská P, Horáková M, Syrovy I, Cinti S and Kopecky J: Expression of the uncoupling protein 1 from the aP2 gene promoter stimulates mitochondrial biogenesis in unilocular adipocytes in vivo. Eur J Biochem 269: 19-28, 2002.
53. Pecqueur C, Alves-Guerra MC, Gelly C, Levi-Meyrueis C, Couplan E, Collins S, Ricquier D, Bouillaud F and Miroux B: Uncoupling protein 2 , in vivo distribution, induction upon oxidative stress, and evidence for translational regulation. J Biol Chem 276: 8705-8712, 2001.

54. Mattiasson G, Shamloo M, Gido G, Mathi K, Tomasevic G, Yi S, Warden CH, Castilho RF, Melcher T, Gonzalez-Zulueta M, Nikolich K and Wieloch T: Uncoupling protein-2 prevents neuronal death and diminishes brain dysfunction after stroke and brain trauma. Nat Med 9: 1062-1068, 2003.

55. Vincent AM, Olzmann JA, Brownlee M, Sivitz WI and Russell JW: Uncoupling proteins prevent glucose-induced neuronal oxidative stress and programmed cell death. Diabetes 53: 726734, 2004.

56. Choi CS, Fillmore JJ, Kim JK, Liu ZX, Kim S, Collier EF, Kulkarni A, Distefano A, Hwang YJ, Kahn M, Chen Y, Yu C, Moore IK, Reznick RM, Higashimori T and Shulman GI: Overexpression of uncoupling protein 3 in skeletal muscle protects against fat-induced insulin resistance. J Clin Invest 117: 1995-2003, 2007.

57. Ho PW, Chu AC, Kwok KH, Kung MH, Ramsden DB and Ho SL: Knockdown of uncoupling protein-5 in neuronal SH-SY5Y cells: Effects on $\mathrm{MPP}^{+}-$induced mitochondrial membrane depolarization, ATP deficiency, and oxidative cytotoxicity. J Neurosci Res 84: 1358-1366, 2006

58. Prabhakaran K, Li L, Mills EM, Borowitz JL and Isom GE: Upregulation of uncoupling protein 2 by cyanide is linked with cytotoxicity in mesencephalic cells. J Pharmacol Exp Ther 314: $1338-1345,2005$

59. Wan CD, Wang CY, Liu T, Cheng R and Wang HB: Alleviation of ischemia/reperfusion injury in ob/ob mice by inhibiting UCP-2 expression in fatty liver. World J Gastroenterol 114: 590-594, 2008.

60. Mills EM, Xu D, Fergusson MM, Combs CA, Xu Y and Finkel T: Regulation of cellular oncosis by uncoupling protein 2 . J Biol Chem 277: 27385-27392, 2002.

61. Diehl AM and Hoek JB: Mitochondrial uncoupling: role of uncoupling protein anion carriers and relationship to thermogenesis and weight control 'the benefits of losing control'. J Bioenerg Biomembr 31: 493-506, 1999. 